\title{
Discrete Radon Transform
}

\author{
GREGORY BEYLKIN
}

\begin{abstract}
This paper describes the discrete Radon transform (DRT) and the exact inversion algorithm for it. Similar to the discrete Fourier transform (DFT), the DRT is defined for periodic vector-sequences and studied as a transform in its own right. Casting the forward transform as a matrix-vector multiplication, the key observation is that the matrix-although very large-has a block-circulant structure. This observation allows construction of fast direct and inverse transforms. Moreover, we show that the DRT can be used to compute various generalizations of the classical Radon transform (RT) and, in particular, the generalization where straight lines are replaced by curves and weight functions are introduced into the integrals along these curves. In fact, we describe not a single transform, but a class of transforms, representatives of which correspond in one way or another to discrete versions of the $\mathrm{RT}$ and its generalizations. An interesting observation is that the exact inversion algorithm cannot be obtained directly from Radon's inversion formula.

Given the fact that the RT has no nontrivial one-dimensional analog, exact invertibility makes the DRT a useful tool geared specifically for multidimensional digital signal processing. Exact invertibility of the DRT, flexibility in its definition, and fast computational algorithm affect present applications and open possibilities for new ones. Some of these applications are discussed in the paper.
\end{abstract}

\section{INTRODUCTION}

$\mathrm{T}$ HE discrete Radon transform (DRT) is a discrete version of the classical Radon transform (RT) [1] and some of its generalizations [2]-[4]. The DRT defined and described in this paper is exactly invertible in an efficient manner. Since RT (and, hence, DRT) have no nontrivial analog in the one-dimensional space, exact invertibility makes DRT a useful tool geared specifically for multidimensional digital signal processing.

Discrete versions of the classical RT are being used in signal processing and there is an extensive literature devoted to this subject. Procedures which are discrete versions of the RT are known as slant stack [5], tau- $P$ transform [6], [7], velocity filtering [8], [9], fan filtration [9], and beam forming [10]. These procedures were successfully used in various applications such as ground roll removal [8], plane wave decomposition [11]-[14], $P-S$ separation [15], interpolation and resampling of data [8], and, also, in procedures such as velocity analysis and beam steering. Some of these applications depend on invertibility of the RT.

There are two major classes of algorithms for inversion of discrete versions of the RT: algebraic reconstruction techniques, and backprojection algorithms based upon

\footnotetext{
Manuscript received January 17, 1986; revised July 10, 1986.

The author is with Schlumberger-Doll Research, Ridgefield, CT 06877 4108 .

IEEE Log Number 8610882 .
}

various discretizations of Radon's inversion formula. We note that in applications mentioned above, algebraic reconstruction techniques (such as iterative and row-action methods [16]-[18] developed for image reconstruction from projections) were not used. Although these procedures can, in principle, provide an exact inversion of the RT, they are not practical in these applications because of the size of the linear system to be solved. As a consequence, all inversion algorithms used, in practice, have been approximate inversions based on Radon's inversion formula. We show, however, that a discrete version of the RT can be inverted only approximately if its inversion is based on a discretization of Radon's formula.

This inversion formula was discovered by J. Radon [1] who was also the first to define the transform as such. The transform itself and inversion formula were later rediscovered for use in different applications which include astrophysics [19] and computer assisted tomography [20] (for a more complete account of applications, see [21]). In seismics, the RT is known as the slant stack or the tau- $P$ transform. The inversion procedure for the RT was rediscovered for the purpose of processing seismograms probably as early as in 1969 [9].

Heuristically, the use of the RT in signal processing can be explained as follows: a seismogram (a multidimensional signal array) can be viewed as a superposition of different events with energy concentrated along straight lines (at least locally). The RT maps these events into points thus allowing identification and separation.

The RT was used by many investigators for transformation and analysis of seismograms [5]-[9], [12]-[14] and, also, for computing synthetic seismograms [11]. An applicable theory of the generalized RT (integration over surfaces or curves with the presence of a weight function) was developed in [2]-[4]. Recently, inversion procedures, known in geophysical literature as migration algorithms, were cast as inversions of the causal generalized RT [22]-[24]. This makes it even more important to look carefully into the problem of exact and fast inversion of discrete versions of the RT and its generalizations.

The approach we adopt in this paper can be illustrated by a parallel with the discrete Fourier transform (DFT). Similar to the DFT, the DRT is defined and studied as a transform in its own right. The DFT, of course, is used to compute direct and inverse continuous Fourier transform integrals, especially using the fast Fourier transform (FFT) algorithm. Similarly, we show how DRT can be used to compute the classical RT, and the inversion procedure for DRT can be used to invert it. Moreover, we 
show that DRT can be used to compute various generalizations of the classical RT, in particular, the generalization where straight lines are replaced by curves and weight functions are introduced into the integrals along those curves. In fact, we describe not a single transform, but a class of transforms. The representatives of this class correspond in one way or another to discrete versions of the $\mathrm{RT}$ and its generalizations.

We treat the inverse problem as a linear algebra problem and reduce it to solving a linear system of equations with a block-circulant matrix. This approach allows us to define the DRT in a consistent way and to obtain a simple inversion procedure. Although this inversion procedure can be classified as an algebraic reconstruction technique, it does not require solving a large linear system. Instead, inversion proceeds frequency by frequency without iterations an requires only solving a small linear system for each frequency. An interesting feature of the DRT is that its inversion procedure is related to the discrete form of Radon's inversion formula, but cannot be derived from this formula directly.

We also note that the results of this paper can be extended to the case of more than one spatial variable.

\section{Classical Radon Transform}

We start by describing the classical RT and one of many possible discretizations of it. We will use it to motivate the definition of DRT and make a comparison of exact inversion to an approximate inversion based on the Radon's inversion formula.

The classical Radon transform of a function $u$ of two variables is a function $R u$ defined on a family of straight lines. The value of the $R u$ on a given straight line is the integral of $u$ along this line. We choose to describe a line in the plane $(t, q)$ as

$$
t=\tau+p q,
$$

where $\tau$ and $p$ are parameters. Using terminology adopted in seismic applications, $\tau$ is intercept time and $p$ is slope. Thinking of $t$ as a time and $q$ as a spatial variable, the function $u(t, q)$ represents a seismogram. The RT in this case is

$$
(R u)(\tau, p)=\int_{-\infty}^{+\infty} u(\tau+p q, q) d q,
$$

and is known as the tau- $P$ transform or slant stack. Radon's inversion formula can be written in operator notation as.

$$
R * K R=I,
$$

where $K$ is a one-dimensional operator (filter) and $R^{*}$ is a dual transform. Given $v(\tau, p)=(R u)(\tau, p)$, it follows from identity (1.2) that the original function can be found as $u=R^{*} K v$. Here the convolutional operator $K$ is given by

$$
(K v)(\tau, p)=\int_{-\infty}^{+\infty}|f| \hat{v}(f, p) e^{-2 \pi i f \tau} d f
$$

where

$$
\hat{v}(f, p)=\int_{-\infty}^{+\infty} v(\tau, p) e^{2 \pi i f \tau} d \tau
$$

The dual transform $R^{*}$ (which also is the adjoint transform in a proper space of functions [25]) is as follows:

$$
\left(R^{*} v\right)(t, q)=\int_{-\infty}^{+\infty} v(t-p q, p) d p
$$

and also is known as the backprojection operator.

Discrete versions of the inversion formula (1.2) are being currently used in a multitude of applications. Here we give an example of the discretization to compare the approach developed later in this paper to the direct use of this formula.

Let a seismogram $u(t, q)$ contain $2 L+1$ traces, i.e., we have $u\left(t, q_{l}\right)$, where $l=0, \pm 1, \cdots, \pm L$. Assuming that $q_{-L}<q_{-L+1}<\cdots<q_{L-1}<q_{L}$, we approximate the integral in (1.1) by

$$
(R u)(\tau, p)=\sum_{l=-L}^{l=L} u\left(\tau+p q_{l}, q_{l}\right) \Delta q_{l},
$$

where $\Delta q_{l}=\left(q_{l+1}-q_{l-1}\right) / 2$ for $l=0, \pm 1, \cdots, \pm(L$ -1 ), and $\Delta q_{L}=q_{L}-q_{L-1}, \Delta q_{-L}=q_{-L+1}-q_{-L}$. To obtain a value of the function $u\left(\tau+p q_{l}, q_{l}\right)$, one might use interpolation in the first variable if necessary. Numerical implementation of the operator $R^{*}$ is completely analogous to (1.5). Assuming that the function $v(\tau, p)$ is known for $2 J+1$ discrete values of the parameter $p$, i.e., given $v\left(\tau, p_{j}\right), j=0, \pm 1, \cdots, \pm J$, we approximate the integral in (1.4) by

$$
\left(R^{*} v\right)(t, q)=\sum_{j=-J}^{j=J} v\left(t-q p_{j}, p_{j}\right) \Delta p_{j},
$$

where $\Delta p_{j}=\left(p_{j+1}-p_{j-1}\right) / 2$ for $j=0, \pm 1, \cdots, \pm(J$ $-1)$, and $\Delta p_{J}=p_{J}-p_{J-1}, \Delta p_{-J}=p_{-J+1}-p_{-J}$. Convolutional operator $K$ in (1.3)-which is the operator of multiplication by $|f|$ in the frequency domain-can be approximated by the so-called Shepp-Logan filter [26], for example.

There are many other possibilities to discretize the RT and the one presented is just an example. This simple discretization is sufficient for us to motivate the definition of the DRT.

\section{Definition OF the DRT}

Let $x_{l}(n)$ be a two-dimensional array, where $-L \leq l$ $\leq L$. For a fixed index $l, x_{l}(n)$ represents a discrete signal which we will refer to as a time series or a seismic trace. We choose an odd number of traces $(2 L+1)$ for convenience. For a fixed time point $n, n=0, \cdots, N-1$, we denote by $x(n)$ the following vector: 


$$
x(n)=\left[\begin{array}{c}
x_{-L}(n) \\
\cdots \\
x_{0}(n) \\
\ldots \\
x_{L}(n)
\end{array}\right] .
$$

We assume that $x(n)$ is defined for all integer $n$ and is a periodic vector sequence with period $N$,

$$
x(n+N)=x(n) .
$$

Assumption (i) here is the same as in the case of the DFT which is defined for periodic sequences.

We consider the following transform:

$$
y(n)=\sum_{m=-M}^{m=M} R_{m} x(n+m),
$$

where $2 M+1 \leq N$ and $R_{m}$ are $(2 J+1) \times(2 L+1)$ transform matrices. In $(2.1), y(n)$ denotes the following periodic vector sequence (with period $N$ ):

$$
y(n)=\left[\begin{array}{c}
y_{-J}(n) \\
\cdots \\
y_{0}(n) \\
\ldots \\
y_{J}(n)
\end{array}\right],
$$

where $y_{j}(n)$ is a two-dimensional array with the index $j$, $-J \leq j \leq J$, representing different slopes. The number $M$ in (2.1) is the number of neighboring vectors on each side of the input vector $x(n)$ involved in computing the output vector $y(n)$.

Definition 1: We call any transform of the form (2.1) a discrete Radon transform.

Remark 1: If $x(n)$ is not periodic, then we can always extend (pad) $x(n)$ by zeros so that it can be considered as a periodic vector sequence with some (sufficiently large) period $N$.

Remark 2: One can argue that Definition 1 is too general, since it is a definition of a shift-invariant multichannel filter with $2 L+1$ inputs and $2 J+1$ outputs. Indeed, Definition 1 is a definition of a class of transforms rather than a single transform. In this paper we consider the general shift-invariant multichannel filter as a generalized discrete RT. To arrive at this point of view, let us describe an extension of the definition of the classical RT so that the transform is defined on an arbitrary parametrized family of geometrical objects. We recall that the classical RT is defined on a family of straight lines; we also consider generalizations where the family of geometrical objects consists of curves. The most general case is, however, when such family consists of arbitrary geometrical objects. The value of such generalized RT on a given geometrical object is an integral over this object.

In the discrete case, these geometrical objects are sub- sets of points of the lattice with a weight coefficient assigned to each point. The family of objects is constructed by invariant shift of such objects. Given a function defined on the lattice, its transform is a new function defined on such family. Its value on a given subset is the sum over this subset of values of the function weighted by corresponding coefficient at each point. Given an arbitrary set of matrices $R_{m}$ in (2.1), the family of the subsets $O(n$, $j$ ) of the lattice parametrized by indexes $n, n=0, \cdots$, $N-1$ and $j, j=-J, \cdots, J$ can be constructed as follows:

$$
\begin{aligned}
O(n, j) & =\left[\{(n+m) \bmod N, j\} \mid\left(R_{m}\right)_{j l} \neq 0, m\right. \\
& =-M, \cdots, M, l=-L, \cdots, L]
\end{aligned}
$$

and weight coefficients are nonzero entries of matrices $R_{m}$. Hence, in the discrete case, one can view matrices $R_{m}$ as templates so that the multichannel filter has the interpretation of generalized RT. Since in applications which motivated this study the point of view of RT is natural, we use the notion of the DRT in this paper.

Remark 3: Two points need to be clarified with respect to this definition. First, we have to show how a discretization of the classical RT (1.1) can be written in the form (2.1). Second, we have to show how to invert the transform in (2.1). The last question will be addressed in the next section. Here we note, that in order for the DRT (2.1) to be an invertible DRT some restrictions on the matrices $R_{m}$ in (2.1) will be imposed.

Let us give a simple example to illustrate the definition and also show how a discretization of the classical RT reduces to (2.1). Let $L=J=1$, so that we have three traces and three slopes. We choose lines so that they pass through the lattice points as illustrated in Fig. 1. The transform matrices in this case are

$$
\begin{aligned}
R_{0} & =\left(\begin{array}{lll}
0 & 1 & 0 \\
1 & 1 & 1 \\
0 & 1 & 0
\end{array}\right), \\
R_{1} & =\left(\begin{array}{lll}
1 & 0 & 0 \\
0 & 0 & 0 \\
0 & 0 & 1
\end{array}\right), \\
R_{-1} & =\left(\begin{array}{lll}
0 & 0 & 1 \\
0 & 0 & 0 \\
1 & 0 & 0
\end{array}\right),
\end{aligned}
$$

and $M=1$. It is clear that transform matrices can be generated in a similar way for any number of traces. In fact, we have as an example of the DRT

$$
\left(R_{m}\right)_{j l}=\delta_{m, j l},
$$

where $\delta$ is the Kronecker symbol with the second subscript being the product of two indexes.

If lines do not pass through lattice points, then inter- 


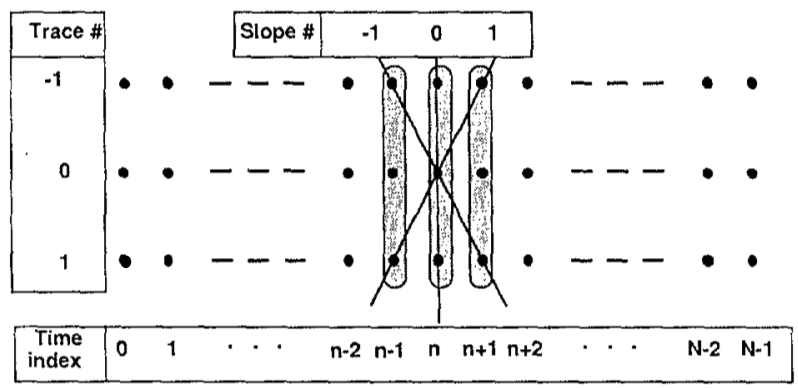

Fig. 1. Illustration showing that the DRT can be written in form (2.1).

polation can be used in between lattice points on each trace. Different interpolation schemes can be used-for example, linear or third order-and lead to different entries for transform matrices $R_{m}$. Interpolation also affects the number $M$ of neighbor-vectors involved. However, we will delay the discussion of these questions until we present an alternative way of defining the transform matrices.

Entries of transform matrices also incorporate information on distances between traces in the form of weight coefficients as can be seen by comparing (1.5) and (2.1). The possibilities of defining transform matrices in different ways will be discussed in greater detail in Sections IV and VII.

To summarize, any specific discretization of (1.1) can always be written in the form (2.1). Moreover, as we will see in Sections IV and VI, much more general transforms than (1.1) yield (2.1) as their discretization.

\section{INVERSION OF THE DRT}

In this section we describe the inversion of the DRT by treating the inverse problem as a linear algebra problem. Here we consider the transform matrices $R_{m}$ given in the time domain while an alternative definition of these matrices in the frequency domain is explained in the next section. If we denote by $x$ the $N \times(2 L+1)$ vector

$$
x=\left(\begin{array}{c}
x(0) \\
x(2) \\
\cdots \\
x(N-1)
\end{array}\right),
$$

then (2.1) can be written as

$$
\boldsymbol{y}=\boldsymbol{R} \boldsymbol{x},
$$

where $R$ is the following block-circulant matrix:

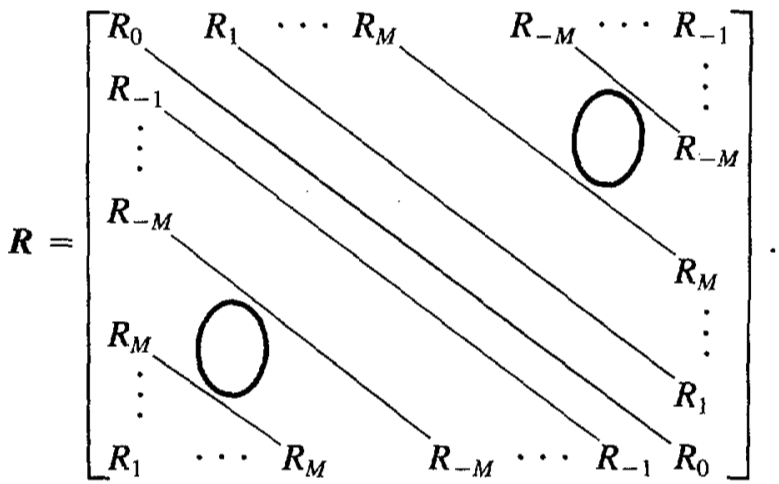

This is the key observation which follows from the periodicity condition (i). (Discussion of properties of the block-circulant matrices can be found in [27], for example.) We also consider the adjoint matrix $\boldsymbol{R}^{*}$

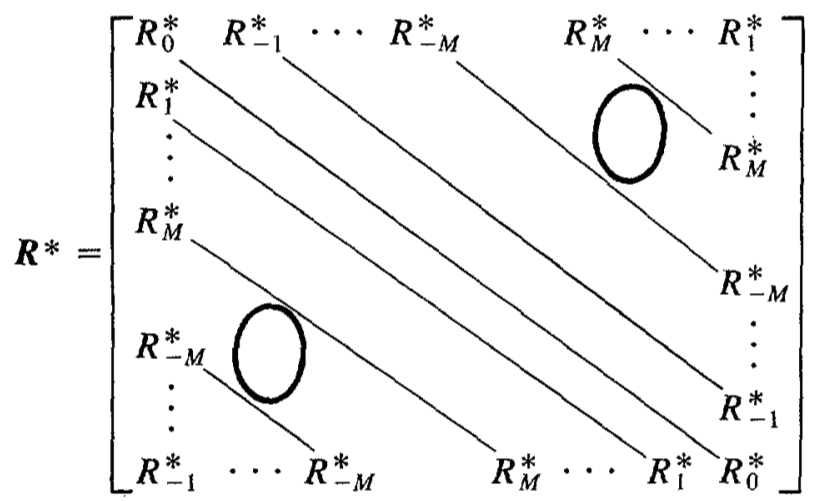

and the transform associated with it

$$
z(n)=\sum_{m=-M}^{m=M} R_{-m}^{*} y(n+m) .
$$

We introduce the matrix $\boldsymbol{H}=\boldsymbol{R} * \boldsymbol{R}$ and the associated transform

$$
z(n)=\sum_{m=-2 M}^{m=2 M} H_{m} x(n+m)
$$

where

$$
H_{m}=\sum_{m^{\prime}=-M}^{m^{\prime}=M-m} R_{m^{\prime}}^{*} R_{m+m^{\prime}}
$$

and

$$
H_{-m}=H_{m}^{*},
$$

for $0 \leq m \leq 2 M$.

Lemma 1: If $z(n)$ is given by (3.2), and $x(n)$ satisfies condition (i) of Section III, then

$$
\hat{z}(k)=\hat{H}(k) \hat{x}(k),
$$

for $k=0,1, \cdots, N-1$, where $\hat{z}(k)$ and $\hat{x}(k)$ are DFT's of $z(n)$ and $x(n)$

$$
\begin{aligned}
& \hat{z}(k)=\sum_{n=0}^{n=N-1} z(n) e^{2 \pi i(n k / N)}, \\
& \hat{x}(k)=\sum_{n=0}^{n=N-1} x(n) e^{2 \pi i(n k / N)},
\end{aligned}
$$

and matrices $\hat{H}(k)$ are as follows:

$$
\hat{H}(k)=\sum_{m=-2 M}^{m=2 M} H_{m} e^{-2 \pi i(m k / N)} .
$$

The proof of Lemma 1 can be found in the Appendix.

Since $x(n)$ is a real vector-sequence $\hat{x}(N-k)=\overline{\hat{x}}(k)$ for $k=1, \cdots,(N-1) / 2$, where the bar denotes complex 
conjugation, it is sufficient to consider (3.4) for $k=0$, $1, \cdots, N / 2$. (Here and elsewhere in the paper, $N / 2$ should be replaced by $(N-1) / 2$ if $N$ is odd.)

Definition 2: We say that the DRT in (2.1) is uniquely invertible within the normalized frequency band $\left[k_{\min } / N\right.$, $\left.k_{\max } / N\right], 0 \leq k_{\min } / N, k_{\max } / N \leq 0.5$, if for all $k=k_{\min }$, $\cdots, k_{\max }$

$$
\operatorname{det} \hat{H}(k) \neq 0 \text {. }
$$

If (ii) is satisfied within the normalized frequency band $\left[k_{\min } / N, k_{\max } / N\right]$, then the inversion procedure for a seismogram band-limited to this band is as follows. Given the transform matrices $R_{m}$, matrices $\hat{H}(k), k=0, \cdots, N / 2$ are precomputed using (3.3) and (3.7) (We will present a better way of computing matrices $\hat{H}(k)$ in Section IV.) Now, given the vector sequence $y(n)$ [the DRT of $x(n)$ ], we compute the following.

1) The adjoint transform of $y(n)$ by using (3.1) to obtain $z(n), n=0, \cdots, N-1$.

2) The FFT of $z(n)$.

3) A solution to a linear $(2 L+1) \times(2 L+1)$ system (3.4) for $k=k_{\min }, \cdots, k_{\max }(\operatorname{det} \hat{H}(k) \neq 0)$ to obtain $\hat{x}(k)$. We set $\hat{x}(k)=0$ outside the frequency band, i.e., for $k=0, \cdots, k_{\min }-1$ and $k=k_{\max }+1, \cdots, N / 2$.

4) Inverse FFT of $\hat{x}(k)$ to obtain $x(n)$.

If (ii) holds for all $k=0, \cdots, N / 2$, then the DRT is invertible for all frequencies. In some important examples, det $\hat{H}(0)=0$, where $k=0$ corresponds to the $\mathrm{dc}$ level of the traces. Indeed, $\hat{x}(0)=\Sigma_{n=0}^{n=N-1} x(n)$, and det $\hat{H}(0)=0$ means that we cannot recover the dc level. In practice, we can always assume that the dc level is zero.

We note that the inversion procedure is based on the identity

$$
\left(R^{*} R\right)^{-1} R^{*} R=I
$$

and steps 2)-4) describe an efficient way of applying the operator $\left(\boldsymbol{R}^{*} \boldsymbol{R}\right)^{-1}$.

\section{FAST DRT}

By considering the transform matrices $R_{m}$ in the frequency domain and making use of the FFT, we show in this section that the DRT can be implemented as a fast algorithm.

Lemma 2: If $y(n)$ is given by (2.1) and $x(n)$ satisfies condition (i) of Section II, then

$$
\hat{y}(k)=\hat{R}(k) \hat{x}(k),
$$

for $k=0,1, \cdots, N-1$, where $\hat{y}(k)$ and $\hat{x}(k)$ are DFT's of $y(n)$ and $x(n)$, respectively.

$$
\begin{aligned}
& \hat{y}(k)=\sum_{n=0}^{n=N-1} y(n) e^{2 \pi i(n k / N)}, \\
& \hat{x}(k)=\sum_{n=0}^{n=N-1} x(n) e^{2 \pi i(n k / N)},
\end{aligned}
$$

and matrices $\hat{R}(k)$ are as follows ${ }^{1}$

$$
\hat{R}(k)=\sum_{m=-M}^{m=M} R_{m} e^{-2 \pi i(m k / N)}
$$

Since $x(n)$ is a real vector-sequence, it is sufficient to consider (4.1) for $k=0,1, \cdots, N / 2$.

Lemma 2 is completely analogous to Lemma 1 , and its proof can be found in the Appendix. This lemma makes the DRT a fast algorithm. Indeed, to compute the DRT of a signal array $x(n)$, we perform the following.

1) FFT of $x(n)$. The number of operations is of order $(2 L+1) \cdot N \cdot \log (N)$.

2) At most, $N / 2$ matrix-vector multiplications (4.1). The number of operations is at most of order $(2 L+1)$. $(2 J+1) \cdot N$.

3) Inverse FFT to obtain $y(n)$. The number of operations is of order $(2 J+1) \cdot N \cdot \log (N)$.

If $N \gg(2 L+1)$ and $N \gg(2 J+1)$ (which is the case in seismic applications), then the algorithm 1)-3) is a fast algorithm. For band-limited signals, step 2) requires even fewer operations. All three steps are extremely well suited for parallel processing since each step contains parallel computations within itself.

The adjoint transform differs from the direct fast DRT only by step 2 ) where matrix $\hat{R}(k)$ is replaced by $\hat{R} *(k)$,

$$
\left(\hat{R}^{*}\right)_{l j}(k)=\overline{\hat{R}}_{j l}(k),
$$

the adjoint to $\hat{R}(k)$. Therefore, steps 1) and 2) in the inversion scheme in Section IV can be replaced by steps 1 ) and 2) of this procedure. Also, it is clear that the matrix $\hat{H}(k)$ in (3.4) can be written as

$$
\hat{H}(k)=\hat{R}^{*}(k) \hat{R}(k),
$$

for all $k$. If the number of slopes is equal to the number of traces (i.e., $\hat{R}(k)$ are square matrices) then $\operatorname{det} \hat{H}(k)=$ $(\operatorname{det} \hat{R}(k))^{2}$. In this case, one can perform the inverse fast DRT by replacing step 2) in algorithm 1)-3) by the solution of a linear system involving matrices $\hat{R}(k)$. - Alternative Definition of the DRT: Since $\hat{R}(N-k)=$ $\overrightarrow{\hat{R}}(k)$, it is sufficient to consider matrices $\hat{R}(k)$ only for $k$ $=0,1, \cdots, N / 2$. Given (4.4), we have

$$
R_{m}=\frac{1}{N} \sum_{k=0}^{k=N-1} \hat{R}(k) e^{2 \pi i(m k / N)}
$$

and, therefore, matrices $\hat{R}(k) k=0,1, \cdots, N / 2$, Define transform matrices $R_{m}$ and the DRT as described in Definition 1 . If $\operatorname{det}\left(\hat{R}^{*}(k) \hat{R}(k)\right) \neq 0$ within some frequency band (Definition 2) then the DRT is uniquely invertible within this frequency band.

In many cases, the direct description of matrices $\hat{R}(k)$ is simpler than that of matrices $R_{m}$. Consider the following matrices for $k=0,1, \cdots, N / 2$ :

$$
\hat{R}_{j l}(k)=e^{-2 \pi i \sigma(j l k / N)} \text {. }
$$

\footnotetext{
${ }^{1}$ The unconventional minus sign in the phase of the exponent in the DFT in (4.4) comes from the choice of the plus sign in definition (2.1) which, in turn, matches the plus sign in definition (1.1) adopted in seismic applications.
} 
It follows from (4.6) that if $\sigma=1$, matrices $R_{m}$ are given by

$$
\left(R_{m}\right)_{j l}=\delta_{m, j l} .
$$

These are the matrices considered in the example in Section II. In the definition (4.7), the description of straight lines is the description of the phase function. The reciprocal of the parameter $\sigma$ in $(4.7), 1 / \sigma$, plays the role of "number of slopes per time step" and eliminates the use of interpolation.

Another choice of matrices $\hat{R}(k)$,

$$
\hat{R}_{j l}(k)=A(j, l) e^{-2 \pi i \phi(j, l) k / N},
$$

defines the DRT for the case with curves. Again, the description of curves is the description of the phase function. Entries of the matrix $A(j, l)$ are interpreted as weight coefficients.

In the most general case, by analogy with $(4.8)$, we can write the transform matrices in the following form:

$$
\hat{R}_{j l}(k)=A(j, l, k / N) e^{-2 \pi i \phi(j, i, k / N)}
$$

Since both the direct and inverse transforms are computed in the frequency domain, the direct description of the matrices in (4.9) is a convenient way of defining the DRT. Matrices $\hat{R}(k)$ in (4.8) describe an important special case since to define the transform in this case it is sufficient to supply just two matrices $\phi(j, l)$ and $A(j, l), j=$ $-J, \cdots, J, l=-L, \cdots, L$.

\section{The DRT and Digrtal Prolate Functions (DPF)}

For general definitions of the DRT as those in (4.8) and (4.9), the matrix $\hat{H}(k)$ in (4.5) is formed numerically and the stability of the inversion is checked for all $k$ of interest within the LU decomposition step of the solution of the corresponding linear system. If $\operatorname{det} \hat{H}(k)$ is numerically small for some $k$, then it means that a nonzero seismogram can be generated containing periodic signals of this frequency and having an almost zero DRT. Inversion in this case becomes numerically unstable. There is a number of approaches to stabilize the inversion. These approaches can be considered as part of the design of the transform and will be treated elsewhere. Here we will discuss the connection of the DRT as defined in (4.7) with the DPF. This connection allows to understand the inversion issue for this particular case.

The DPF play a fundamental role in the analysis of the maximum-energy-concentration problem for periodic functions [28] and are closely related to prolate spheroidal wave functions [29]-[33]. It appears that in a slightly modified form they also play a role in the DRT as defined in (4.7).

Consider a discrete periodic sequence $w(l), w(l+2 L$ $+1)=w(l)$ and the following transform:

$$
\hat{w}_{\alpha}(j)=\sum_{l=-L}^{l=L} w(l) e^{2 \pi i \alpha l j /(2 J+1)},
$$

where $j=0, \pm 1, \cdots, \pm J$. This transform reduces to the ordinary DFT for $\alpha=1$ and $L=J$. We consider now the following problem: given $\alpha$ and $\hat{w}_{\alpha}(j)$ for $j=0$, $\pm 1, \cdots, \pm J$, find $w(l)$. To solve this problem, we apply the normalized adjoint transform (if $\alpha=1$ and $L=J$ this is the inverse DFT)

$$
w^{\prime}\left(l^{\prime}\right)=\frac{\alpha}{2 J+1} \sum_{j=-J}^{j=J} \hat{w}_{\alpha}(j) e^{-2 \pi i \alpha l^{\prime} j /(2 J+1)},
$$

and obtain a linear system

$$
w^{\prime}\left(l^{\prime}\right)=\frac{\alpha}{2 J+1} \sum_{l=-L}^{l=L} w(l) \frac{\sin \pi \alpha\left(l^{\prime}-l\right)}{\sin \pi \alpha \frac{\left(l^{\prime}-l\right)}{2 J+1}} .
$$

Introducing the matrix

$$
P_{l^{\prime} l}=\frac{\alpha}{2 J+1} \frac{\sin \pi \alpha\left(l^{\prime}-l\right)}{\sin \pi \alpha \frac{\left(l^{\prime}-l\right)}{2 J+1}},
$$

this system can be written as

$$
w^{\prime}\left(l^{\prime}\right)=\sum_{l=-L}^{l=L} P_{l^{\prime} l^{\prime}} w(l) .
$$

If the parameter $\alpha$ is bounded and $2 J+1$ goes to infinity, the matrix $P_{l^{\prime} l}$ becomes

$$
P_{l^{\prime} l}^{\infty}=\frac{\sin \pi \alpha\left(l^{\prime}-l\right)}{\pi\left(l^{\prime}-l\right)} .
$$

The matrix $P_{l l}^{\infty}$ in (5.2) has $2 L+1$ positive eigenvalues, and its eigenvectors form an orthonormal system. If we denote by $\psi_{l}^{m}$ the eigenvector corresponding to the eigenvalue $\lambda_{m}$, then

$$
\Psi^{m}(s)=\sum_{l=-L}^{l=L} \psi_{l}^{m} e^{2 \pi i \alpha l s}
$$

are the DPF as described in [28]. In fact, a similar construction of eigenvectors for the matrix $P_{l^{\prime} l}$ leads to the analogs of the DPF for discrete periodic band-limited functions.

For the DRT, the connection with the DPF provides additional insight into the invertibility of the transform. By writing (4.7) as

$$
\hat{R}_{j l}(k)=e^{-2 \pi i \alpha j l /(2 J+1)},
$$

where $\alpha=\sigma k(2 J+1) / N$, and applying the adjoint transform, we obtain

$$
\hat{H}_{l^{\prime} l}(k)=\frac{\sin \pi \alpha\left(l^{\prime}-l\right)}{\sin \pi \alpha \frac{\left(l^{\prime}-l\right)}{2 J+1}} .
$$

This establishes the connection with the DPF since the matrix $\hat{H}_{l^{\prime} l}(k)$ differs from the matrix $P_{l^{\prime} l}$ in $(5.1)$ only by a normalizing factor. By making the number of slopes $2 J$ +1 sufficiently large (while $\alpha=k / k_{0}$ is bounded and $\sigma$ 
$=N / k_{0}(2 J+1)$, where $\left.k_{\min } \leq k_{0} \leq k_{\max }\right)$, estimates of the eigenvalues of the matrix $\hat{H}_{l^{\prime} l}(k)$ can be obtained using those of the eigenvalues of the matrix $P_{l^{\prime} l}^{\infty}$ in (5.2). Hence, the frequency band within which the transform in (4.7) is invertible and the inversion is stable can be estimated.

\section{The DRT and Radon's Inversion Formula}

So far we considered the inversion of the DRT as an algebraic reconstruction technique. In this section we describe the relation of our inversion algorithm to algorithms based on Radon's inversion formula.

Instead of the identity in (3.8), we can write

$$
\boldsymbol{R} *\left(\boldsymbol{R} \boldsymbol{R}^{*}\right)^{-1} \boldsymbol{R}=\boldsymbol{I} \text {. }
$$

This formula produces an alternative inversion procedure. We compute the following.

1a) The FFT of $y(n)$, where $y(n)$ is the DRT of $x(n)$ in (2.1).

2a) A solution to a linear $(2 J+1) \times(2 J+1)$ system

$$
\hat{y}(k)=\hat{\tilde{H}}(k) \hat{w}(k),
$$

for $k=k_{\min }, \cdots, k_{\max }$ to obtain $\hat{w}(k)$. We set $\hat{w}(k)=0$ outside the frequency band, i.e., for $k=0, \cdots, k_{\min }-$ 1 and $k=k_{\max }+1, \cdots, N / 2$. In (6.2), the matrices $\hat{H}(k)$ are as follows:

$$
\hat{\hat{H}}(k)=\hat{R}(k) \hat{R} *(k) .
$$

3a) Inverse FFT of $\hat{w}(k)$ to obtain $w(n)$.

4a) The adjoint transform (3.1) of the sequence $w(n)$ to obtain $x(n)$.

We note that if the adjoint transform is computed by the procedure described in Section IV, then step 3a) is not necessary.

The significance of considering (6.1) becomes clear when the inversion procedure 1a)-4a) is compared to the inversion based on Radon's inversion formula (1.2). It follows from (6.1) and (1.2) that the operator $\boldsymbol{R}$ corresponds to the discretization of the continuous Radon transform (1.1), the operator $R^{*}$ to the dual transform (backprojection) (1.4) and, therefore, the operator $\left(\boldsymbol{R} \boldsymbol{R}^{*}\right)^{-1}$ must correspond to a discretization of the onedimensional filter $K(1.3)$.

The one-dimensional filter $K$ in (1.3) can be implemented as an operator solving the linear system in (6.2) only if all the matrices $\hat{H}(k)$ are diagonal. This is because whatever discretization is used to implement the operator $K$ in (1.3) it cannot produce nondiagonal matrices $\tilde{H}(k)$ since the operator $K$ is applied to each trace independently of other traces. It is easy to check that, in (6.3), at least some of the matrices $\hat{\tilde{H}}(k)$ are nondiagonal, and, therefore, the exact inversion procedure described here does not follow from discretizations of Radon's inversion formula. In other words, the discrete transform must be considered as such to obtain its exact inversion procedure since it cannot be deduced from Radon's inversion formula by means of discretization.
Inversion formula (6.1) also implies the discrete Parseval's identity. In the continuous case, Parseval's identity for the classical Radon transform was discussed in [25]. We define the inner product for seismograms in the time-space domain as

$$
\left(\boldsymbol{x}, \boldsymbol{x}^{\prime}\right)=\sum_{n=0}^{n=N-1} \sum_{l=-L}^{l=L} x_{i}(n) x_{l}^{\prime}(n)
$$

and in the tau- $P$ domain as

$$
\left[\boldsymbol{y}, \boldsymbol{y}^{\prime}\right]=\sum_{n=0}^{n=N-1} \sum_{j=-J}^{j=J} y_{j}(n) y_{j}^{\prime}(n) .
$$

Given the inner product in the time-space domain, the norm of a seismogram is

$$
\|\boldsymbol{x}\|=\left(\sum_{n=0}^{n=N-1} \sum_{l=-L}^{l=L} x_{l}^{2}(n)\right)^{1 / 2} .
$$

It follows from (6.1) that

$$
(x, x)=\left(R^{*}\left(\boldsymbol{R} \boldsymbol{R}^{*}\right)^{-1} \boldsymbol{R} x, x\right)=\left[\left(\boldsymbol{R} \boldsymbol{R}^{*}\right)^{-1} \boldsymbol{R} x, \boldsymbol{R} x\right],
$$

and, therefore, that

$$
\|x\|^{2}=\left[\left(R R^{*}\right)^{-1} y, y\right],
$$

where $\boldsymbol{y}=\boldsymbol{R} \boldsymbol{x}$ is the DRT of the seismogram $\boldsymbol{x}$. The matrix $\boldsymbol{R} \boldsymbol{R}^{*}$ is always self-adjoint and nonnegative definite. If the transform is invertible for all frequencies then this matrix is positive definite. In this case, the negative powers of $\boldsymbol{R} \boldsymbol{R}^{*}$ are well defined and we obtain

$$
\|\boldsymbol{x}\|^{2}=\left\|\boldsymbol{K}^{1 / 2} \boldsymbol{y}\right\|^{2},
$$

where $\boldsymbol{K}^{1 / 2}=\left(\boldsymbol{R} \boldsymbol{R}^{*}\right)^{-1 / 2}$. The relation (6.4) is Parseval's identity for the DRT. If the transform is invertible within a frequency band, then (6.4) holds for seismograms bandlimited to this band.

\section{Application of the DRT to Computation of Generalized Radon Transforms}

If instead of (1.1) we consider the generalized Radon transform,

$$
(R u)(\tau, p)=\int_{-\infty}^{+\infty} u(\tau+\phi(p, q), q) a(p, q) d q,
$$

i.e., an integral along curves $t=\tau+\phi(p, q)$ with a weight function $a(p, q)$, the same considerations apply. Discretization of (7.1) can be written in the form (2.1) and, therefore, the inversion procedure for the DRT can be used. The expression (7.1) [and (1.1) as a particular case of (7.1)] can be written in the following form:

$$
\begin{aligned}
& (R u)(\tau, p) \\
& \quad=\int_{-\infty}^{+\infty} d f e^{-2 \pi i f r} \int_{-\infty}^{+\infty} d q \hat{u}(f, q) a(p, q) e^{-2 \pi i f \phi(p, q)} .
\end{aligned}
$$


One can see now that the expression in (4.8) is a discrete analog of the kernel in the inner integral in (7.2). If we consider an even more general transform

$$
\begin{aligned}
& (R u)(\tau, p) \\
& =\int_{-\infty}^{+\infty} d f e^{-2 \pi i f \tau} \int_{-\infty}^{+\infty} d q \hat{u}(f, q) a(p, q, f) e^{-2 \pi i f \phi(p, q, f)},
\end{aligned}
$$

then (4.9) provides a discrete version of its kernel. In all of these cases, the discrete formulation falls within the definition of the DRT. Therefore, the algorithm for computing the DRT and the inverse transform can be utilized.

The geometrical objects over which we integrate need not be simple curves (see remark 2 of Section II). These objects can be of any shape as long as we can discretize the integral in the form (2.1). This opens a number of new possibilities in digital processing of seismograms as well as image processing.

In signal processing of seismograms there are situations where it might be advantageous to integrate along curves or strips bounded by curves, hyperbolas, for example. Also, the ability to compute integrals as those in (7.2) and (7.3) with the DRT algorithm combined with results in [22]-[24] might help to cast inversion and migration procedures as a signal processing algorithm employing the DRT as the main computational engine.

In image processing, integration over different shapes is used for pattern recognition. The classical RT is known as the Hough transform [34] in these applications, and the generalized RT in this case can be viewed as a process of "comparing" a given pattern produced by transform matrices $R_{m}$ with the signal array. The tau- $P$ domain in this case is the domain of responses. If, in this domain, responses for different shapes can be isolated, then the inversion procedure makes it possible to separate patterns in the original domain.

Since weight functions can be adequately implemented in transform matrices, we can choose these weights for each given seismogram separately. This can be achieved by using some statistical measure on the data set. For example, we can use semblance criteria [35] to compute weights. This leads to the nonlinear transform as follows.

1) Given a multidimensional signal array, we choose transform matrices $R_{m}$ in (2.1) [or $\hat{R}(k)$ in (4.4)] according to some statistical measure computed for this array and so that the transform is invertible within the frequency band of interest.

2) Given the transform matrices, we apply the transform to the signal array and obtain generalized tau- $P$ representation of this array. We can then analyze this representation, separate events, apply windows, filters, etc.

3) Now we apply the inverse transform. The inversion procedure obtains the original representation without losing any information due to inaccuracies in reconstruction. All modifications are due to operations performed at step 2).

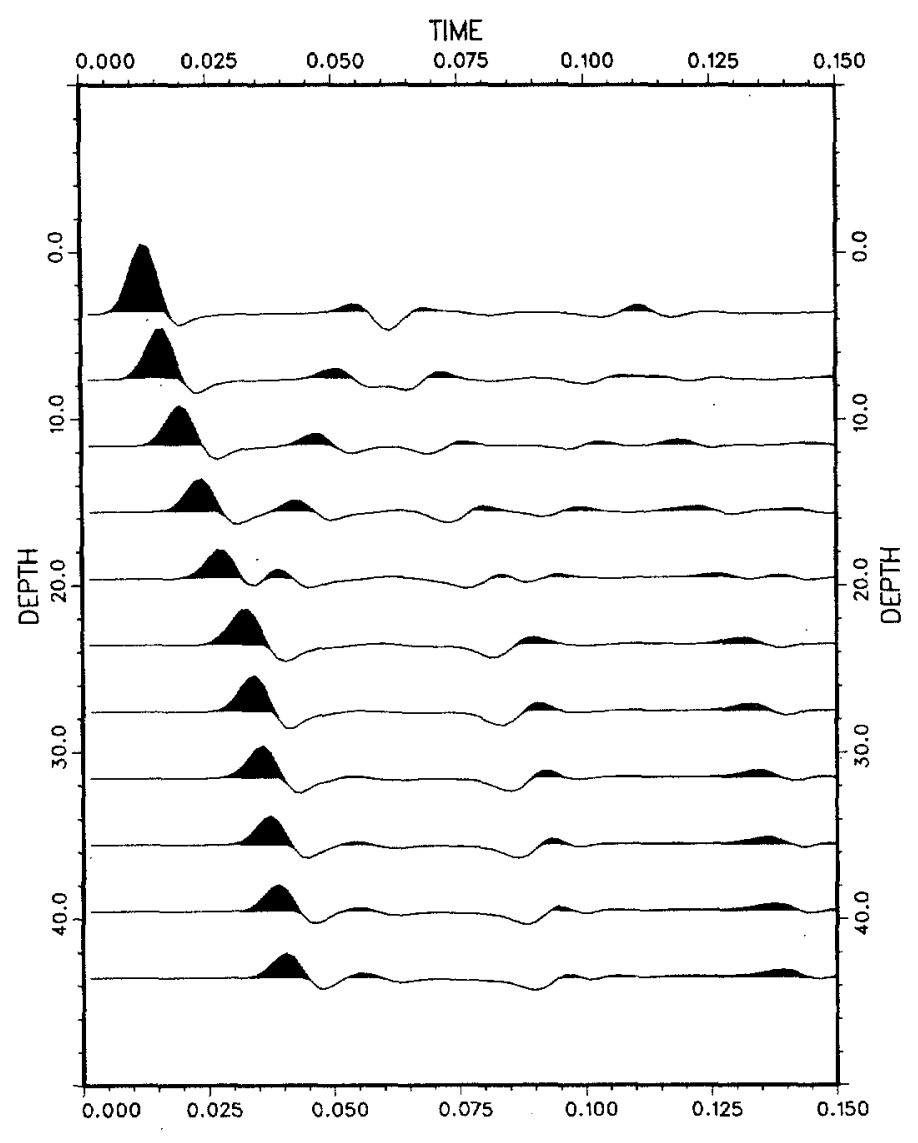

Fig. 2. Example 1. Synthetic VSP section.

\section{EXAMPLES}

We present two examples to illustrate the use of the DRT. We choose synthetic VSP (vertical seismic profile) seismograms as signal arrays. A VSP seismogram is a recording of the seismic signal in a borehole with the source on the surface. One of the signal processing tasks in this context is the separation of the upgoing waves from the downgoing waves.

In the first example, we explain the role of the tau- $P$ representation. We apply the DRT (4.7) to the synthetic VSP data set in Fig. 2. In this example, the number of time samples is $N=100$, and the number of traces $2 L+$ $1=11$. We choose the number of slopes to be $2 J+1=$ 11 and the parameter $\sigma=1$. The result of the transform is shown in Fig. 3. The lower portion of the tau- $P$ representation in Fig. 3 corresponds to the downgoing waves (positive slopes) and the upper portion corresponds to the upgoing waves (negative slopes). Inverse transform gives us back the original seismogram in Fig. 2 and, therefore, the tau- $P$ representation of the seismogram contains as much information as the original seismogram. However, in this example, the accuracy of the reconstruction is low (3 significant digits). This is caused by small determinants of the corresponding linear systems for low frequencies.

To avoid this problem in the second example, we uniformly shift the spectrum of the VSP seismogram in Fig. 4 to higher frequencies, and incorporate such shift into the DRT. In this example, the number of time samples is 


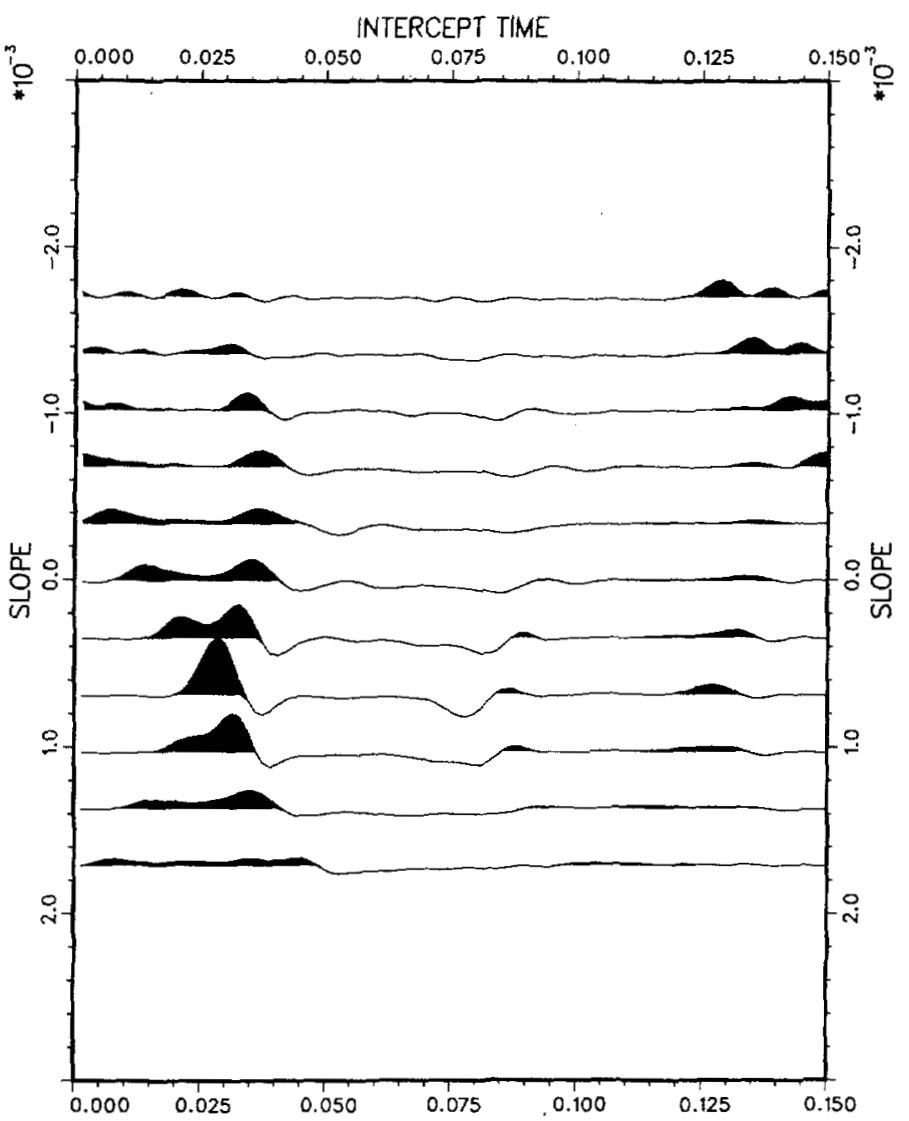

Fig. 3. The tau-P representation of data in Fig. 2. The DRT is described in (4.7).

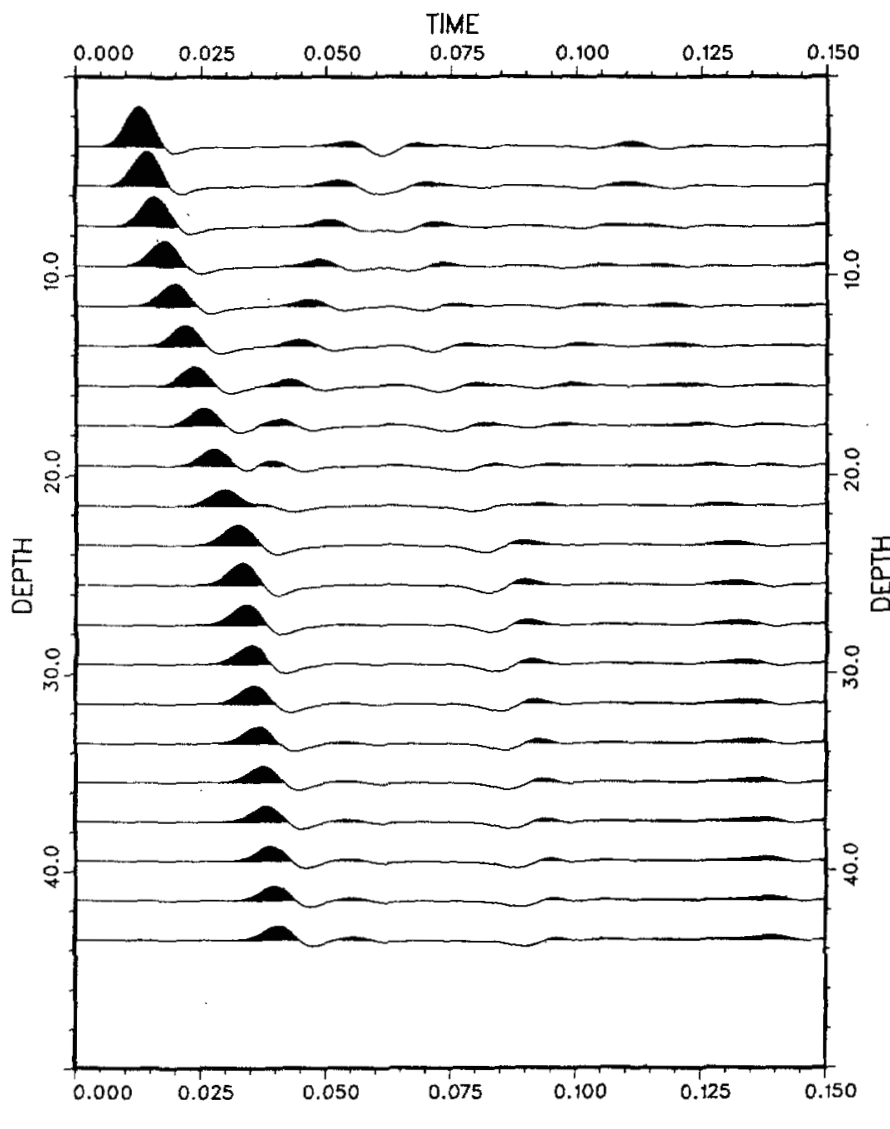

Fig. 4. Example 2. Synthetic VSP section.

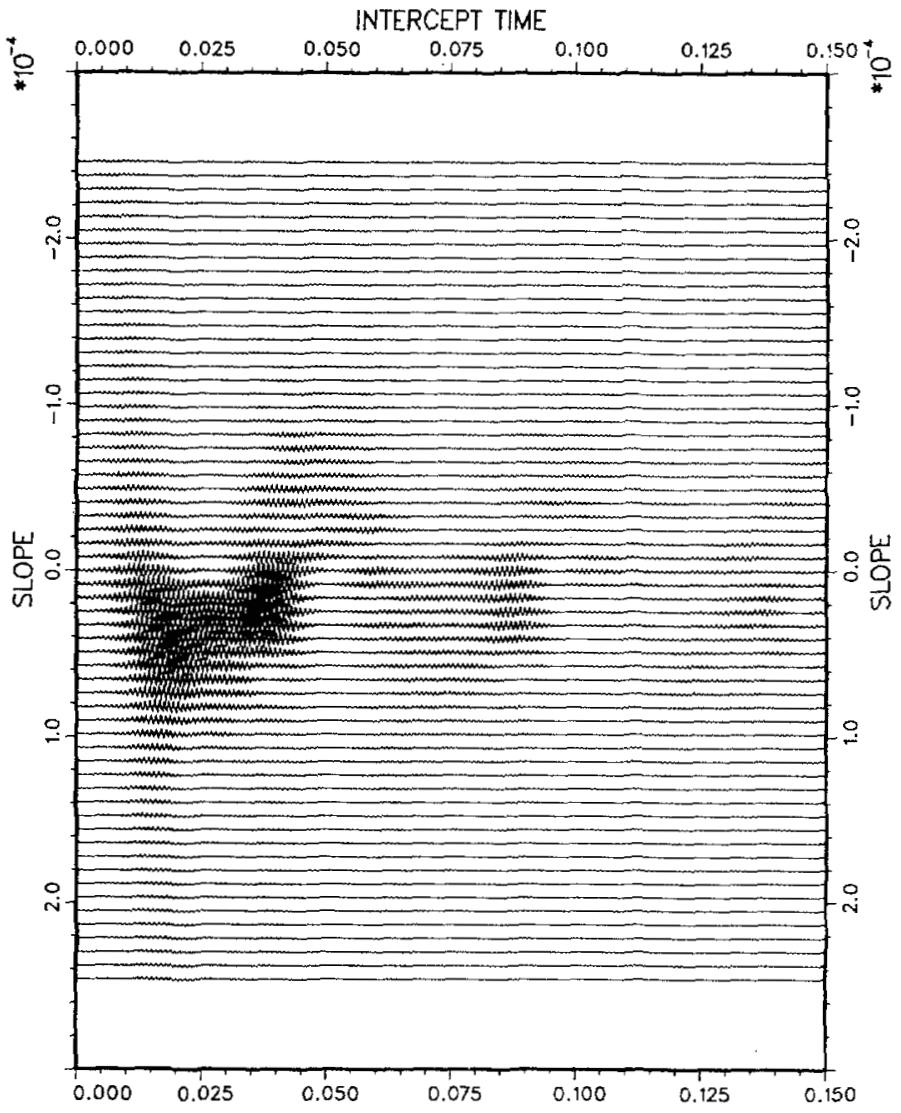

Fig. 5. The tau- $P$ representation of data in Fig. 4 . The DR'T is described in $(7.1)$.

$N=600$, and the number of traces is $2 L+1=21$. We choose the number of slopes to be $2 J+1=61$. The shift of the spectrum is incorporated into the DRT by the following transform matrices:

$$
\hat{R}_{j l}(k)=e^{-2 \pi i \sigma j l\left(k+k_{s}\right) / N},
$$

for $k=k_{\min }, \cdots, k_{\max }$, where $k_{\min }=1$ and $k_{\max }=20$. The shift of the spectrum is described by the parameter $k_{s}$ and, in our example, $k_{s}=199$. The parameter $\sigma$ we choose to be $\sigma=N /(2 J+1) k_{s}$. Applying the DRT in (8.1), we obtain the tau- $P$ representation shown in Fig. 5. If we apply the inverse DRT, we obtain the original data set.

To demonstrate the application of the DRT to the velocity filtering (upgoing and downgoing waves separation in this case), we mask a part of the tau- $P$ representation. The mask is shown in Fig. 6 and is chosen to remove one upgoing event in seismogram in Fig. 4. The effect of such masking can be seen by comparing Fig. 4 and Fig. 7. The seismogram in Fig. 7 is obtained by applying the inverse DRT to the masked tau- $P$ representation in Fig. 6 . The event is "surgically" removed with a very good preservation of the amplitude information. In any case, all changes in the amplitude are due to the effects of the mask itself. To underscore the robustness of masking in the tau$P$ domain, the mask was obtained by merely setting to zero all the values within the window in Fig. 6. We note that the difficulty in distinguishing between effects of the 


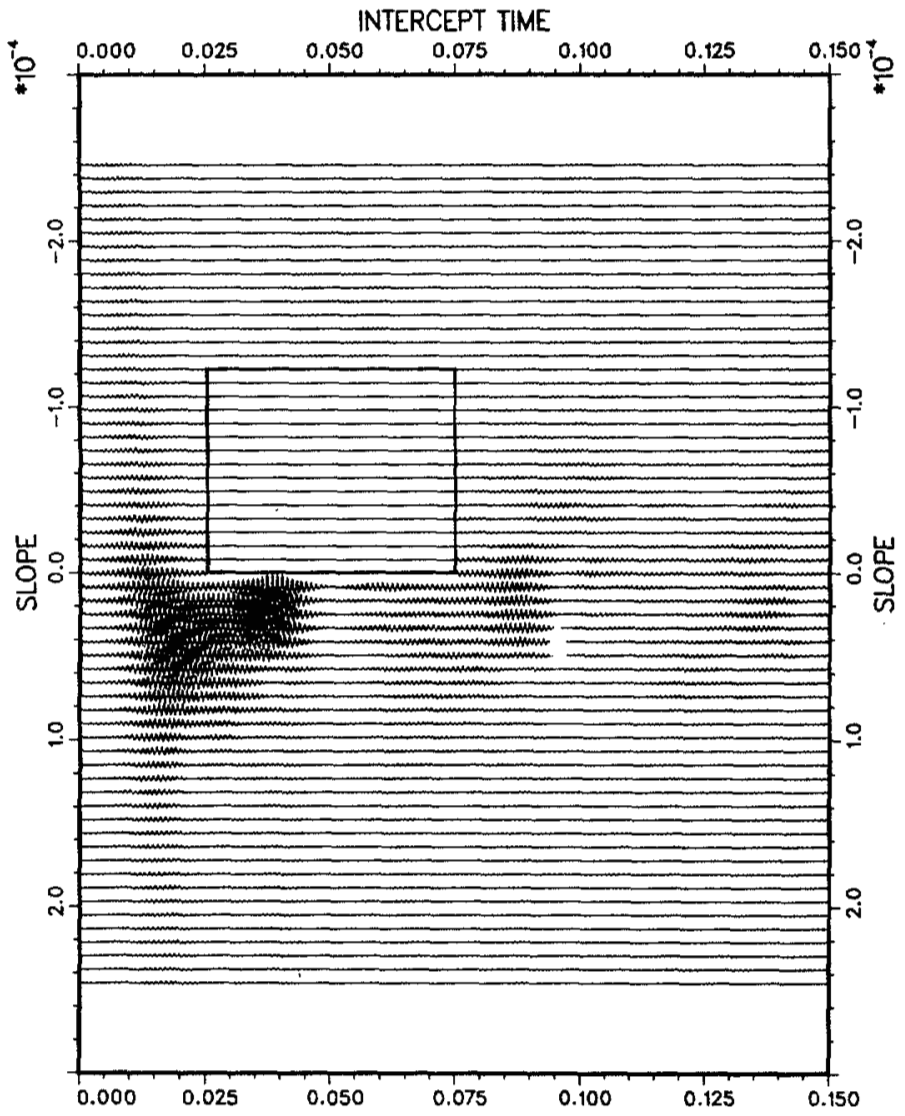

Fig. 6. Example of mask in the tau- $P$ domain to remove one upgoing event in seismogram in Fig. 4.

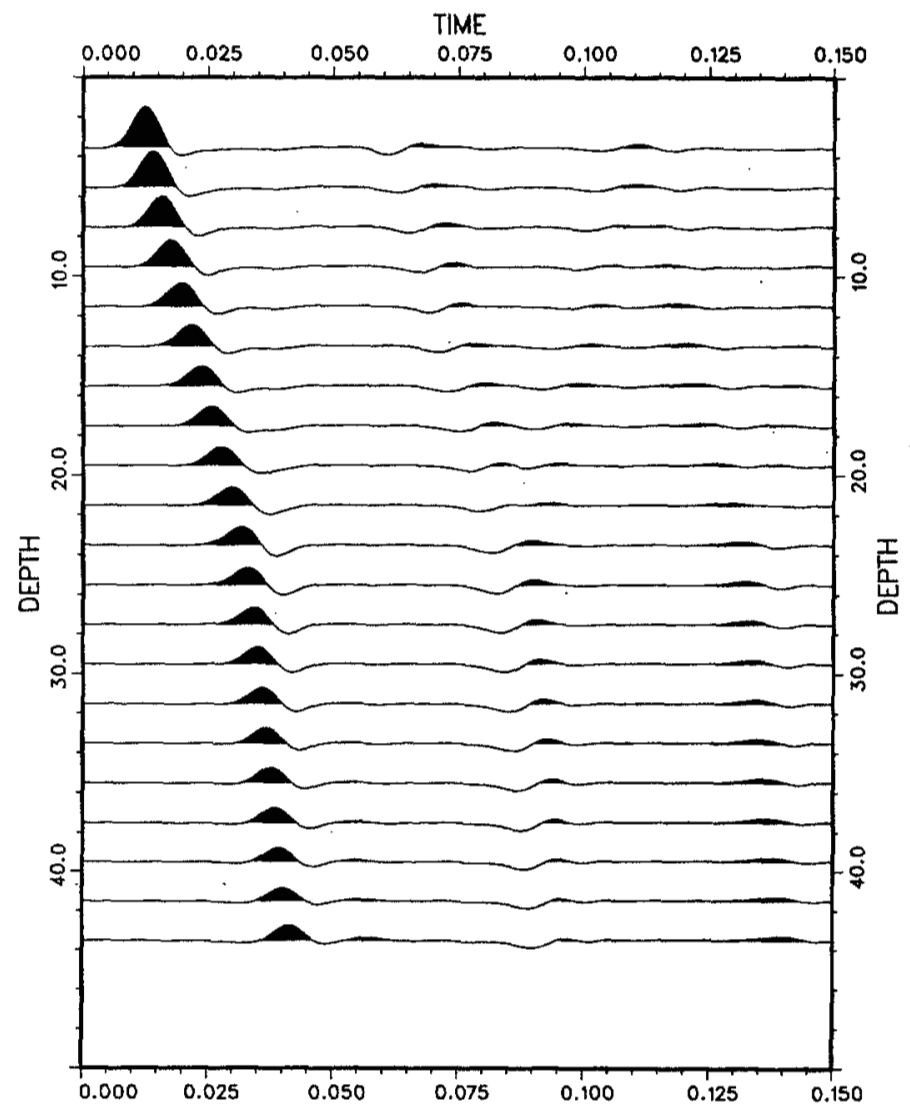

Fig. 7. Reconstruction by applying the inverse DRT to the masked tau- $P$ representation in Fig. 6. The effect of masking can be seen by comparison to Fig. 4. mask and the approximate inversion was a problem in using the tau- $P$ representation for the velocity filtering.

\section{APPENDIX}

Lemma 1 and Lemma 2 are essentially similar. Their proof is elementary. We use the notation of Lemma 1. Applying the DFT to both sides of (3.2), we obtain

$$
\hat{z}(k)=\sum_{m=-2 M}^{m=2 M} H_{m} \sum_{n=0}^{n=N-1} x(n+m) e^{2 \pi i(n k / N)},
$$

or

$$
\hat{z}(k)=\sum_{m=-2 M}^{m=2 M} H_{m} e^{-2 \pi i(m k / N)} \sum_{n=m}^{n=N+m-1} x(\tilde{n}) e^{2 \pi i(\tilde{n} k / N)} .
$$

Condition (i) implies that

$$
\sum_{n=0}^{n=m-1} x(\tilde{n}) e^{2 \pi i(\tilde{n} k / N)}=\sum_{\tilde{n}=N}^{\tilde{n}=N+m-1} x(\tilde{n}) e^{2 \pi i(n k / N)},
$$

for $m \geq 1$. A similar identity holds for $m \leq-1$ and, hence,

$$
\hat{z}(k)=\sum_{m=-2 M}^{m=2 M} H_{m} e^{-2 \pi i(m k / N)} \sum_{n=0}^{\tilde{n}=N-1} x(\tilde{n}) e^{2 \pi i(\tilde{n} k / N)}
$$

which is exactly (3.4).

\section{ACKNOWLEDGMENT}

The author wishes to thank the reviewers for their constructive suggestions and valuable comments.

\section{REFERENCES}

[1] J. Radon, "Uber die Bestimmung von Funktionen durch ihre Integralwerte langs gewisser Mannigfaltigkeiten," Berichte Sachsische Acadamie der Wissenschaften, Leipzig, Math.-Phys. Kl., vol. 69, pp. 262-267, 1917.

[2] G. Beylkin, "Generalized Radon transform and its application," Ph.D. dissertation, New York Univ., New York, NY, 1982.

[3] - , "Inversion of the generalized Radon transform," in Proc. SPIE, Inverse Optics, vol. 413, pp. 32-40, 1983.

[4] _ - "The inversion problem and applications of the generalized Radon transform," Commun. Pure Appl. Math., vol. 37, pp. 579-599, 1984.

[5] P. S. Schultz and J. F. Claerbout, "Velocity estimation and downward continuation by wavefront synthesis," Geophysics, vol. 43, pp. 691-714, 1978.

[6] E. N. Bessonova, V. M. Fishman, V. Z. Ryaboyan, and G. A. Sitnikova, "The tau method for the inversion of travel times-I. Deep seismic sounding data," J. Roy. Astron. Soc., vol. 36, pp. 377-398, 1974.

[7] E. N. Bessonova, V. N. Fishman, M. G. Schoriman, and G. A. Sitnikova, "The tau method for the inversion of travel times-II. Earthquake data," J. Roy. Astron. Soc., vol. 46, pp. 87-108, 1976.

[8] R. H. Tatham, J. Keeney, and I. Noponen, "Application of the tau$P$ transform (slant stack) in processing seismic reflection data," preprint of this paper presented at the 52nd Annu. Meet. SEG, Dallas, TX, 1982.

[9] S. A. Nakhamkin, "Fan filtration," Izv., Earth Phys., vol. 11, pp. 24-35, 1969.

[10] D. E. Dudgeon and R. M, Mersereau, Multidimensional Digital Signal Processing. Englewood Cliffs, NJ: Prentice-Hall, 1984.

[11] C. H. Chapman, "A new method for computing synthetic seismograms," Geophys. J. Roy. Astron. Soc., vol. 54, pp. 481-518, 1978.

[12] - "Generalized Radon transforms and slant stacks," Geophys. J. Roy. Astron. Soc., vol. 66, pp. 445-453, 1981.

[13] R. A. Phinney, K. R. Chowdhury, and L. N. Frazer, "Transforma- 
tion and analysis of record sections," $J$. Geophys. Res., vol. 86, no. B1, pp. 359-377, 1981 .

[14] P. L. Stoffa, P. Buhl, J. B. Diebold, and W. Friedemann, “Direct mapping of seismic data to the domain of intercept time and ray parameters, a plane wave decomposition," Geophysics, vol. 46, pp. 255-267, 1981

[15] A. J. Devaney and M. L. Oristaglio, "A plane-wave decomposition for elastic wavefields applied to the separation of $P$-waves and $S$-waves in vector seismic data,"' Geophysics, vol. 51, no. 2, pp. 419-423, 1986.

[16] G. T. Herman, A. Lent, and S. W. Rowland, "ART: Mathematics and applications," J. Theoret. Biol., vol. 42, pp. 1-32, 1973.

[17] K. A. Dines and R. J. Lytle, "Computerized geophysical tomography," Proc. IEEE, vol. 67, no. 7, pp. 1065-1073, 1979.

[18] Y. Censor, "Row-action methods for huge and sparse systems and their applications,"'SIAM Rev., vol. 23, no. 4, pp. 444-464, 1981.

[19] R. N. Bracewell, "Strip integration in radio astronomy," Aust. J. Phys., vol. 9, pp. 198-201, 1956.

[20] A. M. Cormack, "Representation of a function by its line integrals, with some radiological applications," J. Appl. Phys., vol. 34, pp. 2722-2727, 1963 .

[21] S. R. Deans, The Radon Transform and Some of Its Applications. New York: Wiley-Interscience, 1983.

[22] G. Beylkin, "Imaging of discontinuities in the inverse scattering problem by inversion of a causal generalized Radon transform," $J$. Math. Phys., vol. 26, Jan. 1985.

[23] D. Miller, M. Oristaglio, G. Beylkin, "A new formalism and old heuristic for seismic migration," extended Abstract for 54th Annu. Int. Meet. Soc. Exploration Geophys., 1984.

[24] _ - "A new slant on seismic imaging: Classical migration and integral geometry," to appear in Geophysics.

[25] D. Ludwig, "The Radon transform on Euclidian space," Comm. Pure Appl. Math., vol. 19, pp. 49-81, 1966.

[26] L. A. Shepp and B. F. Logan, "The Fourier reconstruction of a head section," IEEE Trans. Nucl. Sci., vol. NS-21, pp. 24-43, 1974.

[27] A. S. Willsky, Digital Signal Processing and Control and Estimation Theory. Cambridge, MA: M.I.T. Press, 1979.

[28] A. Papoulis and M. S. Bertran, "Digital filtering and prolate functions," IEEE Trans. Circuit Theory, vol. CT-19, pp. 674-681, 1972.

[29] D. S. Slepian and H. O. Pollack, "Prolate spheroidal wave functions, Fourier analysis, and uncertainty-I," Bell Syst. Tech. J., vol. 40, no. 1, pp. 43-64, 1961.
[30] H. J. Landau and H. O. Pollack, "Prolate spheroidal wave functions, Fourier analysis, and uncertainty-II,' Bell Syst. Tech. J., vol. 40, no. 1, pp. $65-84,1961$.

[31] _ - "Prolate spheroidal wave functions, Fourier analysis, and uncertainty-III," Bell Syst. Tech. J., vol. 41, no. 4, pp. 1295-1336, 1962

[32] D. S. Slepian, "Prolate spheroidal wave functions, Fourier analysis, and uncertainty-IV," Bell Syst. Tech. J., vol. 43, no. 6, pp. 3009$3058,1964$.

[33] — , "Prolate spheroidal wave functions, Fourier analysis, and uncertainty-V: The discrete case,"'Bell Syst. Tech. J., vol. 57, no. 5, pp. $1371-1430,1978$.

[34] A. Iannino and S. D. Shapiro, "A survey of the Hough transform and its extension for curve detection," in Proc. IEEE Comput. Soc. Pattern Recogn. Image Processing, 1978, pp. 32-38.

[35] C. V. Kimball and T. L. Marzetta, "Semblance processing of borehole acoustic array data," Geophysics, vol. 49, no. 3, pp. 274-281, 1984.

Gregory Beylkin was born in Leningrad, USSR, on March 16, 1953. He received the Diploma (equivalent to the M.S. degree) in mathematics from Leningrad University in 1975 and the Ph.D. degree in mathematics from New York University (Courant Institute of Mathematical Sciences-CIMS), New York, NY, in 1982.

From 1976 to 1979 he worked at the Research Institute of Ore Geophysics, Leningrad. He emigrated from the USSR in 1979. In 1982-1983 he was an Associate Research Scientist at CIMS. Since 1983 he has been a Member of the Professional Staff at Schlumberger-Doll Research, Ridgefield, CT. His current research is concerned with theory of wave propagation and multidimensional inverse scattering problems. He is the author and coauthor of more than 20 papers.

Dr. Beylkin is a member of the Society for Industrial and Applied Mathematics (SIAM), American Mathematical Society (AMS), and the New York Academy of Sciences. 